\title{
Comparison of Volume Status in Asymptomatic Haemodialysis and Peritoneal Dialysis Outpatients
}

\author{
Eugenia Papakrivopoulou John Booth Jennifer Pinney \\ Andrew Davenport
}

UCL Centre for Nephrology, Royal Free Hospital, University College London Medical School, London, UK

\author{
Key Words \\ Bioimpedance $\cdot$ Peritoneal dialysis $\cdot$ Haemodialysis $\cdot$ Extracellular water $\cdot$ Total body water
}

\begin{abstract}
Background: The majority of haemodialysis (HD) patients gain weight between dialysis sessions and thereby become volume overloaded, whereas peritoneal dialysis (PD) is a more continuous technique. Cardiovascular mortality and hypertension is increased with both treatment modalities. We therefore wished to compare volume status in PD and HD to determine whether PD patients are chronically volume overloaded, as a risk factor for cardiovascular mortality. Study Design, Setting and Participants: We retrospectively audited 72 healthy HD patients and 115 healthy PD patients attending a university hospital dialysis centre for routine outpatient treatment, who had multi-frequency bioimpedance measurements of extracellular water to total body water (ECW/TBW). Results: The groups were well matched for age, sex, weight and ethnicity, PD patients had greater urine output $[1,075(485-1,613)$ vs. $42.5(0-1,020) \mathrm{ml} / \mathrm{day}, \mathrm{p}<0.001]$, but there was no difference in antihypertensive prescription (63.5 vs. 76.4\%), mean arterial blood pressure (post-dialysis $101.6 \pm 1.5 \mathrm{~mm} \mathrm{Hg}$ vs. pre-dialysis $102 \pm 2.4 \mathrm{~mm} \mathrm{Hg}$ ), although post-dialysis arterial blood pressure was lower than in PD patients $(96.4 \pm 3.1 \mathrm{~mm} \mathrm{Hg}, \mathrm{p}<0.05)$. The ratio of ECW/TBW fell after HD (pre-dialysis $0.394 \pm 0.001$ vs. post-dialysis $0.389 \pm 0.004, p<$ $0.001)$ and was similar in the PD group to the group before HD $(0.393 \pm 0.001)$, and greater than that in the group after HD $(p<0.001)$. ECW/TBW was greater than the normal reference range in $30 \%$ PD patients, $28 \%$ patients before HD and 20\% patients after HD. Conclusions: Overhydration is common in healthy stable PD outpatients, and ECW volumes in PD patients are not dissimilar to those of pre-dialysis HD patients. The role of chronic volume overload as a risk factor for cardiovascular disease needs further investigation.




\section{Introduction}

Despite the many technological advances in both haemodialysis and peritoneal dialysis over the years $[1,2]$, the mortality of chronic kidney disease patients treated by dialysis (CKD5d) remains disappointingly high, with some studies reporting 5-year survival rates similar to those of patients with solid organ malignancies [3, 4]. Although CKD5d patient mortality varies between countries, mortality rates do appear to be falling [5].

Haemodialysis and peritoneal dialysis treatments differ fundamentally in terms of one being an intermittent therapy, with marked changes in volume status and fluctuating blood pressure, whereas the other modality is continuous. Despite these differences, mortality is similar with both modalities [6], with only a few reports suggesting a marginal benefit for those patients choosing peritoneal dialysis [7]. As with haemodialysis, cardiovascular disease remains the commonest cause of death for peritoneal dialysis patients [8].

Although there are many potential cardiovascular risk factors for the dialysis patient, including both conventional (including smoking, hypertension, diabetes mellitus, hypercholesterolaemia and left ventricular hypertrophy) and CKD-specific risk factors (including anaemia, hyperphosphataemia and hyperhomocysteinaemia), there has been recent interest in the role of hypervolaemia as a cardiovascular risk factor [9]. Haemodialysis patients accumulate fluid between dialysis sessions, and more recently with the introduction of bioimpedance assessments it is now recognised that peritoneal dialysis patients may also be volume overloaded $[10,11]$. We therefore decided to audit volume status in haemodialysis and peritoneal dialysis patients attending our university hospital dialysis centre, to compare volume status in these two groups.

\section{Patients and Methods}

We studied 72 healthy adult haemodialysis patients attending our university hospital outpatient haemodialysis units for thrice weekly haemodialysis treatments, using high-flux haemodialysers, and 115 healthy peritoneal dialysis patients attending for routine outpatient assessment, with $33 \%$ prescribed hypertonic glucose exchanges (22.7 g/l glucose) and $>95 \%$ icodextrin. All patients had bioimpedance assessments, these were made prior to the midweek dialysis session and then 20 min after dialysis for the haemodialysis cohort to allow for re-equilibration [12], and following a standard peritoneal equilibrium test for the peritoneal dialysis group [13]. As such, pregnant patients, those with amputations and cardiac pacemakers or defibrillators were excluded from the study as bioimpedance measurements were not made. Volume and body composition measurements were made using a direct multifrequency bioelectrical impedance (MF-BIA) analysis method using an eight hand and feet tactile electrode system (Biospace in body 720, Seoul, South Korea) [14]. Height was measured by a standard wall mounted measure (Sigmeas 1, Doherty signature range, www.mediclick.co.uk).

Serum biochemistry samples were analysed with a standard multi-channel biochemical analyser (Roche Integra, Roche Diagnostics, Lewes, UK), using the bromcresol green method for albumin determination; N-terminal pro-brain natriuretic peptide (NT-proBNP) was measured by immunoassay (ECLIA Roche Diagnostics, GMBH, Mannheim, Germany) and NT-proBNP samples were taken after the midweek dialysis session [15]. 24-hour urine collections were analysed to determine urine volume and sodium content. Routine chest X-rays and standard two-dimensional transthoracic echocardiograms (Philips IE33, Philips Medical Systems, The Netherlands, www.healthcare.philips.com) were reviewed. 
Table 1. Peritoneal dialysis patients before haemodialysis and a minimum of $20 \mathrm{~min}$ after haemodialysis to allow for redistribution

\begin{tabular}{lccc}
\hline & PD & pre-HD & post-HD \\
\hline Patients, $\mathrm{n}$ & 115 & 72 & 72 \\
Weight, kg & $70.1 \pm 1.4$ & $68.8 \pm 1.7$ & $66.9 \pm 1.7$ \\
BMI & $25.7 \pm 0.4$ & $25.3 \pm 0.7$ & $24.7 \pm 0.7$ \\
BSA, m ${ }^{2}$ & $1.77 \pm 0.02$ & $1.74 \pm 0.03$ & $1.72 \pm 0.03$ \\
SBP, mm Hg & $140.7 \pm 2.3$ & $147.2 \pm 3.3$ & $138.0 \pm 3.0$ \\
DBP, mm Hg & $81.8 \pm 1.4$ & $79.4 \pm 0.4$ & $75.6 \pm 0.3^{* *}$ \\
MAP, mm Hg & $101.6 \pm 1.5$ & $102.1 \pm 2.4$ & $96.4 \pm 2.1^{*}$ \\
ICW, $1 / 1.73$ m $^{2}$ & $20.92 \pm 0.27$ & $21.64 \pm 0.37$ & $21.05 \pm 0.39^{*}$ \\
ECW, $1 / 1.73$ m $^{2}$ & $13.68 \pm 0.19$ & $14.04 \pm 0.23$ & $13.21 \pm 0.21$ \\
ECW/TBW & $0.393 \pm 0.001$ & $0.394 \pm 0.001$ & $0.389 \pm 0.004^{* * *}$ \\
Fat mass, kg & $21.9 \pm 0.9$ & & $19.5 \pm 1.3$ \\
Skeletal muscle mass, kg & $26.1 \pm 0.6$ & & $25.6 \pm 0.8$ \\
Body fat, \% & $30.6 \pm 0.9$ & & $26.5 \pm 1.5$ \\
Waist:hip ratio & $0.94 \pm 0.01$ & & $0.95 \pm 0.01$ \\
\hline
\end{tabular}

$\mathrm{PD}=$ Peritoneal dialysis patients; $\mathrm{HD}=$ haemodialysis patients; $\mathrm{BMI}=$ body mass index; $\mathrm{BSA}=$ body surface area; $\mathrm{SBP}=$ systolic blood pressure; $\mathrm{DBP}=$ diastolic blood pressure; $\mathrm{MAP}=$ mean arterial blood pressure; ICW $=$ intracellular water; ECW $=$ extracellular water. ${ }^{*} \mathrm{p}<0.05$ vs. PD; ${ }^{* *} \mathrm{p}<0.01$ vs. PD; ${ }^{* * *} \mathrm{p}<0.001$ vs. PD.

Ethical approval was granted by the local ethical committee as audit and clinical service development.

\section{Statistical Analysis}

Statistical analysis was by Student's $t$ test for normally distributed data and Mann-Whitney $U$ test for nonparametric data (GraphPad Prism version 4.0, San Diego, Calif., USA). In addition $\chi^{2}$ analysis with correction for small numbers and analysis of variance with postanalysis correction were also performed using SPSS software for Windows version 15.0 (SPSS Inc., Chicago, Ill., USA). Data are expressed as mean \pm SE, median and interquartile range, or percentages. Statistical significance was taken at or below the $5 \%$ level.

\section{Results}

There was no difference in age between the haemodialysis cohort $(55.0 \pm 0.0$ years $)$ and the peritoneal dialysis cohort ( $55.4 \pm 1.4$ years), or sex distribution (50\% male vs. $44.4 \%$ male, respectively). However, there were more diabetics in the haemodialysis cohort ( 36.1 vs. $\left.20 \% ; \chi^{2} 5.14, \mathrm{p}=0.023\right)$, and more patients were prescribed insulin ( $20.8 \mathrm{vs.} 9.1 \%$; $\left.\chi^{2} 4.61, \mathrm{p}=0.31\right)$. However, there were no differences in dialysis vintage [median 22 (4.5$39)$ vs. 25 (3-43) months], or race ( 54.2 vs. $57.4 \%$ Caucasoids). As expected the haemodialysis patients had weight reduction with dialysis, but there was no difference in weight compared to the peritoneal dialysis cohort (table 1). Body composition was similar in the two groups (table 1). Urine output was greater in the peritoneal dialysis group [median $1,075(485-1,613)$ vs. $42.5(0-1,010) \mathrm{ml} /$ day, $\mathrm{p}<0.001]$, as was urinary sodium excretion $[57$ (13-99) vs. $0(0-61) \mathrm{mmol} / \mathrm{day}, \mathrm{p}<0.001]$, and more were prescribed diuretics (77.4 vs. $\left.38.9 \% ; \chi^{2} 4.95, \mathrm{p}=0.026\right)$. However, similar numbers were prescribed anti-hypertensives 
Fig. 1. ECW/TBW ratio in haemodialysis (HD) patients attending for midweek haemodialysis session (pre- and post-HD values), and peritoneal dialysis (PD) patients with peritoneal dialysate drained out. ${ }^{*} \mathrm{p}<0.05 ;{ }^{* *} \mathrm{p}<0.01$ vs. PD patients.

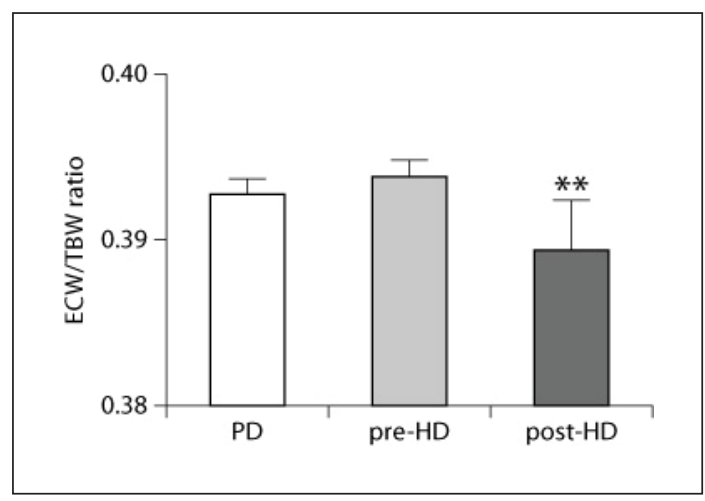

Table 2. Peritoneal dialysis patients before and after haemodialysis

\begin{tabular}{lccc}
\hline & PD & pre-HD & post-HD \\
\hline Sodium, mmol/l & $138 \pm 0.3$ & $139.8 \pm 0.35^{* * *}$ & $140.7 \pm 0.23^{* * *}$ \\
Calcium, mmol/l & $2.29 \pm 0.02$ & $2.28 \pm 0.04$ & $2.15 \pm 0.02^{* *}$ \\
Glucose, mmol/l & $6.4 \pm 0.3$ & $7.6 \pm 1.0$ & $6.8 \pm 0.2$ \\
Albumin, g/l & $39.7 \pm 0.9$ & $40.5 \pm 0.5$ & $43.6 \pm 0.7^{* *}$ \\
CRP, mg/l & $3(1-10)$ & $5(2-13)^{*}$ & \\
PTH, pmol/l & $23.4(13.8-40.3)$ & $20.2(8.8-44.6)$ & $251(118-606)$ \\
NT-proBNP, pmol/l & $242(90-688)$ & & \\
\hline
\end{tabular}

$\mathrm{PD}=$ Peritoneal dialysis patients; $\mathrm{HD}=$ haemodialysis patients; Calcium = corrected serum calcium; $\mathrm{CRP}=\mathrm{C}$-reactive protein; PTH = parathyroid hormone. ${ }^{*} \mathrm{p}<0.05 \mathrm{vs} . \mathrm{PD} ;{ }^{* *} \mathrm{p}<0.01 \mathrm{vs} . \mathrm{PD} ;{ }^{* *} \mathrm{p}<0.001$ vs. PD.

(63.5 vs. 76.4\%), and the median number of anti-hypertensives prescribed was not different [1 (0-2) vs. $1(1-2)]$.

Just $>30 \%$ of the peritoneal dialysis group had a ratio of extracellular water to total body water (ECW/TBW) above the normal reference range of 0.36-0.40, which was similar to the pre-haemodialysis cohort (fig. 1). The ECW/TBW ratios were higher in the peritoneal dialysis group compared to those after haemodialysis (table 1). Serum sodium was lower in the peritoneal dialysis group, and C-reactive protein higher in the haemodialysis cohort, with just under half the haemodialysis cohort dialysing with central venous access catheters. NTproBNP levels (table 2), cardiac dimensions on chest X-ray and transthoracic echocardiography were not different (table 3 ).

\section{Discussion}

It is readily accepted that the majority of patients attending for outpatient haemodialysis are volume overloaded and require ultrafiltration during a haemodialysis session. In our study of asymptomatic stable peritoneal dialysis patients attending for routine assessments of peritoneal dialysis adequacy and transport status (who were not thought to be clinically volume overloaded), volume status assessed by MF-BIA was similar to that of patients prior to haemodialysis, supporting earlier smaller studies [16]. Thus, despite greater urine output 
Table 3. Transthoracic echocardiographic examinations of haemodialysis and peritoneal dialysis patients

\begin{tabular}{lcc}
\hline & \multicolumn{1}{l}{ PD } & HD \\
\hline CTR & $0.47 \pm 0.007$ & $0.49 \pm 0.008$ \\
LVEDD, cm & $4.69 \pm 0.07$ & $4.55 \pm 0.08$ \\
LVES, cm & $3.08 \pm 0.07$ & $3.15 \pm 0.1$ \\
IVSD, cm & $1.2 \pm 0.02$ & $1.2 \pm 0.02$ \\
PWD, cm & $1.13 \pm 0.12$ & $1.14 \pm 0.02$ \\
LASD, cm & $4.0 \pm 0.14$ & $3.95 \pm 0.09$ \\
Ejection fraction, \% & $57.7 \pm 0.9$ & $56.2 \pm 1.3$ \\
RVSP, mm Hg & $25.9 \pm 1.4$ & $27.7 \pm 1.2$ \\
Diastolic dysfunction & $1(1-1)$ & $1(0-1)$ \\
Systolic dysfunction & $0(0-0)$ & $1(0-1)$
\end{tabular}

$\mathrm{PD}=$ Peritoneal dialysis patients; $\mathrm{HD}=$ haemodialysis patients; CTR = cardiothoracic ratio from departmental chest X-ray; LVEDD = left ventricular end-diastolic diameter; LVES = left ventricular end-systolic diameter; IVSD = intraventricular septal diameter; PWD = posterior wall diameter; LASD = left atrial end-systolic diameter; RVSP = estimated right ventricular end-systolic pressure; Diastolic/Systolic dysfunction = left ventricular diastolic and systolic dysfunction.

and urinary sodium excretion peritoneal dialysis patients are similarly volume overloaded as haemodialysis patients.

The results of MF-BIA depend upon the resistance and reactance to the passage of an electrical current, and our MF-BIA device has been validated in both healthy controls [17, 18] and haemodialysis [14] and peritoneal dialysis patients [19]. MF-BIA is affected by body composition which changes with age, sex and race [20], and as such it has been suggested that volumes should be corrected for height [21] or body surface area [22]. Volume overload in incident peritoneal dialysis patients is known to adversely determine outcome; however, it is not generally recognised that peritoneal dialysis patients are chronically volume overloaded [23]. However, we did not find any differences in ECW, or when corrected for height or body surface area with the haemodialysis group, either before or after haemodialysis. Other reports have reported that ECW is greater in peritoneal dialysis patients compared to after dialysis [24]. This was a smaller study of predominantly Caucasoid patients with fewer diabetics. However, an increased ECW/TBW ratio can also be caused by a reduction in TBW due to a loss of intracellular water (ICW). Nearly all our peritoneal dialysis patients used icodextrin, which can increase plasma osmolality [25], due to the accumulation of metabolites [26], and could therefore potentially lead to water removal from and reduction in ICW. Another possible cause of a reduction is ICW protein energy malnutrition [27]. However, in our study both NT-proBNP values, a marker of extracellular volume expansion [15], and ECHO cardiography dimensions were not different between the peritoneal and haemodialysis groups, in keeping with the changes in ECW/TBW which were due to ECW expansion, and not ICW loss. This is supported by body composition which was similar between the groups in terms of skeletal muscle and fat mass. As such, the ECW/TBW in the peritoneal dialysis patients cannot be explained by a loss of ICW and supports ECW expansion.

The lower serum albumin and sodium in the peritoneal dialysis group could be due to volume overload and dilution, and one previous study showed that serum albumin concentrations increased following deliberate ultrafiltration [28]. The lower sodium, however, could also be an artefact of laboratory error [29] due to the usage of icodextrin [26, 30]. 
As expected, blood pressure fell after haemodialysis [31], but pre-dialysis blood pressure was not different compared to the peritoneal dialysis cohort. However, both post-dialysis mean arterial pressure and diastolic blood pressure were lower than those in the peritoneal dialysis group. Both groups had similar antihypertensive medication prescription, although more peritoneal dialysis patients were prescribed loop diuretics, reflecting greater urine volumes. In keeping with blood pressure, transthoracic echocardiography assessments of cardiac size were similar between the groups, as were NT-proBNP levels. It could be speculated that as NT-proBNP is degraded by the kidney, then levels should have been lower in the peritoneal dialysis group due to better residual renal function.

After peritonitis [32], ultrafiltration failure and clinically apparent volume overload are the next most common causes of peritoneal dialysis technique failure [33]. Our study shows that stable asymptomatic peritoneal dialysis patients are equally volume overloaded when compared to haemodialysis patients. As such, this chronic volume overload may account for the prevalence of hypertension and increased cardiovascular death rate in this group of patients [33].

\section{Acknowledgement}

The study was funded by Royal Free Hospital.

\section{Disclosure Statement}

The authors have no conflict of interest.

\section{References}

1 Davenport A: Membrane designs and composition for hemodialysis, hemofiltration and hemodiafiltration: past, present and future. Minerva Urol Nefrol 2010;62:29-40.

-2 Davenport A: Can advances in haemodialysis machine technology prevent intradialytic hypotension? Seminars Dialysis 2009;22:231-236.

-3 Robinson BM, Port F: International haemodialysis patient outcomes comparisons revisited: the role of practice patterns and other factors. Clin J Am Soc Nephrol 2009;4(suppl 1):S12-S17.

-4 Lang K, Korn JR, Lee DW, Lines LM, Earle CC, Menzin J: Factors associated with improved survival among older colorectal cancer patients in the US: a population-based analysis. BMC Cancer 2009;9: 227.

-5 Ansell D, Roderick P, Steenkamp R, Tomson CR: UK Renal Registry 12th Annual Report (December 2009): Chapter 7: Survival and causes of death of UK adult patients on renal replacement therapy in 2008: national and centre-specific analyses. Nephron Clin Pract 2010;115(suppl 1):c117-c144.

-6 Mehrotra R, Chiu YW, Kalantar-Zadeh K, Bargman J, Vonesh E: Similar outcomes with hemodialysis and peritoneal dialysis in patients with end-stage renal disease. Arch Intern Med 2011;171:110118.

>7 Weinhandl ED, Foley RN, Gilbertson DT, Arneson TJ, Snyder JJ, Collins AJ: Propensity-matched mortality comparison of incident haemodialysis and peritoneal dialysis patients. J Am Soc Nephrol 2010;21:499-506.

-8 Michels WM, Verduijn M, Boeschoten EW, Dekker FW, Krediet RT, NECOSAD Study Group: Similar survival on automated peritoneal dialysis and continuous ambulatory peritoneal dialysis in a large prospective cohort. Clin J Am Soc Nephrol 2009;4:943-949.

-9 Agarwal R: Hypervolaemia is associated with increased mortality among haemodialysis patients. Hypertension 2010;56:512-517. 
10 Davenport A, Willicombe M: Comparison of fluid status in patients treated by different modalities of peritoneal dialysis using multi-frequency bioimpedance. Int J Artif Organs 2009;32:779-786.

-11 Davenport A, Willicombe MK: Does diabetes mellitus predispose to increased fluid overload in peritoneal dialysis patients? Nephron Clin Pract 2010;114:c60-c66.

-12 Booth J, Pinney J, Davenport A: The effect of vascular access modality on changes in fluid content in the arms as determined by multifrequency bioimpedance. Nephrol Dial Transplant 2011;26:227231.

-13 Davenport A, Willicombe MK: Hydration status does not influence peritoneal equilibration test ultrafiltration volumes. Clin J Am Soc Nephrol 2009;4:1207-1212.

-14 Fürstenberg A, Davenport A: Comparison of multifrequency bioelectrical impedance analysis and dual-energy X-ray absorptiometry assessments in outpatient hemodialysis patients. Am J Kid Dis 2011;57:123-129.

15 Booth J, Pinney J, Davenport A: N-terminal proBNP - marker of cardiac dysfunction, fluid overload, or malnutrition in haemodialysis patients? Clin J Am Soc Nephrol 2010;5:1036-1040.

-16 Devolder I, Verleysen A, Vijt D, Vanholder R, Van Biesen W: Body composition, hydration, and related parameters in haemodialysis versus peritoneal dialysis patients. Perit Dial Int 2010;30:208-214.

17 Thomas EL, Frost G, Harrington T, Bell JD: Validation on 'in Body' bioelectrical impedance by whole body MRI. Laboratory report 2001.

-18 Bedogni G, Malavolti M, Severi S, Poli M, Mussi C, Fantuzzi AL, Battistini N: Accuracy of an eightpoint tactile-electrode impedance method in the assessment of total body water. Eur J Clin Nutr 2002;56:1143-1148.

-19 Fürstenberg A, Davenport A: Assessment of body composition. Am J Nephrol 2011;33:150-156.

-20 Woodrow G, Devine Y, Cullen M, Lindley E: Application of bioelectrical impedance to clinical assessment of body composition in peritoneal dialysis. Perit Dial Int 2007;27:496-502.

-21 Van der Kerkhof J, Hermans M, Beerenhout C, Konings C, Van der Sande FM, Kooman JP: Reference values for multifrequency bioimpedance analysis in dialysis patients. Blood Purif 2004;22:301-306.

-22 Woodrow G, Oldroyd B, Wright A, Coward WA, Truscott JG: The effect of normalization of ECW volume as a marker of hydration in peritoneal dialysis patients and controls. Perit Dial Int 2005; 25(suppl 3):S49-S51.

-23 Chen W, Guo LJ, Wang T: Extracellular water/intracellular water is a strong predictor of patient survival in incident peritoneal dialysis patients. Blood Purif 2007;25:260-266.

- 24 Chen YC, Lin CJ, Wu CJ, Chen HH, Yeh JC: Comparison of extracellular volume and blood pressure in haemodialysis and peritoneal dialysis patients. Nephron Clin Pract 2009;113:c112-c116.

- 25 Ota K, Akiba T, Nakao T, Nakayama M, Maeba T, Park MS, Tranaeus A, Yorioka N, Icodextrin Study Group: Peritoneal ultrafiltration and serum icodextrin concentration during dialysis with $7.5 \%$ icodextrin solution in Japanese patients. Perit Dial Int 2003;23:356-361.

-26 García-López E, Lindholm B: Icodextrin metabolites in peritoneal dialysis. Perit Dial Int 2009;29: 370-376.

27 Woodrow G: Extracellular water expansion: part of the malnutrition-inflammation-atherosclerosis syndrome? Perit Dial Int 2006;26:566-570.

28 Jones CH, Wells L, Stoves J, Farquhar F, Woodrow G: Can a reduction in extracellular fluid volume result in increased serum albumin in peritoneal dialysis patients? Am J Kidney Dis 2002;39:872-875.

- 29 Davenport A: Interdialytic weight gain in diabetic haemodialysis patients and diabetic control as assessed by glycated haemoglobin. Nephron Clin Pract 2009;113:c33-c37.

-30 Davies SJ, Woodrow G, Donovan K, Plum J, Williams P, Johansson AC, Bosselmann HP, Heimbürger O, Simonsen O, Davenport A, Tranaeus A, Divino Filho JC: Icodextrin improves the fluid status of peritoneal dialysis patients: results of a double-blind randomized controlled trial in patients randomized to glucose or icodextrin for the long exchange. J Am Soc Nephrol 2003;14:2338-2344.

- 31 Davenport A: Intradialytic complications during haemodialysis. Haemodial Int 2006;10:162-167.

- 32 Davenport A: Peritonitis remains the major clinical complication of peritoneal dialysis: the London, UK, peritonitis audit 2002-2003. Perit Dial Int 2009;29:297-302.

- 33 Jager KJ, Merkus MP, Dekker FW, Boeschoten EW, Tijssen JG, Stevens P, Bos WJ, Krediet RT: Mortality and technique failure in patients starting chronic peritoneal dialysis: results of The Netherlands Cooperative Study on the Adequacy of Dialysis. NECOSAD Study Group. Kidney Int 1999;55:14761485. 